(C) Dereito Vol.27, n01:153-176 (Xaneiro-Xuño, 2018) • ISSN 1132-9947

\title{
LE SOCIETÀ MISTE DI GESTIONE DI SERVIZI PUBBLICI LOCALI IN ITALIA: L'INNOVAZIONE NORMATIVA E IL MODELLO EUROPEO
}

\author{
Mixed Companies Managing Local Public Services in Italy: Regulatory \\ Innovation and the European Model
}

DOI: http://dx.doi.org/10.15304/dereito.27.1.4566.5577

\author{
SANDRA ANTONIAZZI \\ Professore Aggregato di Diritto Amministrativo \\ Università di Roma "Tor Vergata" \\ sandra.antoniazzi@uniroma2.it
}

\section{Sommario}

La società mista per la gestione di servizi pubblici è uno schema giuridico noto e rinvenibile in diversi ordinamenti nazionali come forma di collaborazione pubblico-privata e, quindi, di partenariato tra enti locali e operatori economici; le soluzioni tecniche, soprattutto per la scelta del socio privato, inizialmente dipendevano dalle tradizioni differenziate dei singoli ordinamenti secondo un percorso, ancora attuale e non sempre lineare, tra il diritto pubblico e il diritto privato con obiettivi comuni. I riferimenti ad alcune interessanti esperienze nazionali e l'analisi dello schema italiano di società mista consente di acquisire il quadro generale delle questioni di fondo anche in relazione alle riforme degli enti territoriali nel contesto della crisi finanziaria ed economica. Nell'ordinamento italiano, la disciplina si presenta particolarmente intricata nella sua evoluzione fino al D. Lgs. 19 agosto 2016, n. 175, sulle società a partecipazione pubblica, e non facilmente riconducibile ad una definita politica dei servizi pubblici locali, come emerge dai numerosi tentativi di adattamento normativo alle indicazioni dell'UE e dalle riforme interne volte alla riduzione della spesa pubblica per gli enti locali. In particolare, le società di economia mista sono riconducibili al partenariato pubblico-privato istituzionalizzato, distinto dal PPP contrattuale, soluzioni richiamate in vari documenti ufficiale europei, senza elaborare una definizione normativa generale, ma delineando comunque un modello europeo comune in via di attuazione anche mediante la nuova disciplina dei contratti pubblici.

Parole chiave: Società miste, Gestione di servizi pubblici locali, enti locali e operatori economici, Partenariato pubblico privato istituzionalizzato, Modello europeo.

\section{Abstract}

The mixed company for the management of public utilities is a well-known legal scheme that can be found in various national systems as an example of public-private partnership and, therefore, of partnership between local authorities and economic operators. The technical solutions, above all for

https://orcid.org/0000-0003-1365-8082

Recibido: 05/04/2018. Aceptado: 03/05/2018. 
the choice of the private partner, initially depended on the differentiated traditions of the single systems according to a path, still current and not always linear, between public law and private law with common objectives. The references to some interesting national experiences and the analysis of the Italian scheme of a mixed company allow to acquire the general framework of the basic issues also in relation to the reforms of the local authorities in the context of the financial and economic crisis. In the Italian legal system, the discipline is particularly intricate in its evolution up to the D. Lgs. 19 August 2016, n. 175, on companies with public participation, and not easily attributable to a defined policy of local public services, as emerges from the numerous attempts at regulatory adaptation to EU law and guidelines and internal reforms aimed at reducing public spending for local authorities. In particular, the mixed economy societies represent the institutionalised public-private partnership, distinct from the contractual PPP, solutions referred to in various official European documents, without elaborating a general regulatory definition, but nevertheless delineating a common European model being implemented also through the new discipline of public contracts.

Keywords: Mixed companies, Managing local public utilities, Local authorities and Economic Operators, Institutionalised Public Private Partnership, European Model.

\section{INDICE}

1. LE SOCIETÀ MISTE PER LA GESTIONE DI SERVIZI PUBBLICI LOCALI E LA LORO DIFFUSIONE NEL REGNO UNITO, IN FRANCIA, SPAGNA E GERMANIA. - 2. LE SOCIETÀ MISTE NELL'ORDINAMENTO ITALIANO E L'INTRICATO QUADRO NORMATIVO PRIMA DELLA LEGGE DELEGA 7 AGOSTO 2015, N. 124. - 3. L'INNOVAZIONE NORMATIVA: L'ART. 17 DEL DECRETO LEGISLATIVO 19 AGOSTO 2016, N. 175, SULLE SOCIETÀ A PARTECIPAZIONE MISTA PUBBLICO-PRIVATA. - 4. LA SOCIETÀ MISTA COME ESPRESSIONE DI PPPI E LA PROMOZIONE DEL MODELLO EUROPEO IN UN CONTESTO DI CARENZA DI RISORSE FINANZIARIE E ORGANIZZATIVE DEGLI ENTI LOCALI. - 5 . LE CONCLUSIONI DELL'INDAGINE. - 6. BIBLIOGRAFIA.

\section{SUMMARY}

1. MIXED COMPANIES FOR THE MANAGEMENT OF LOCAL PUBLIC SERVICES AND THEIR DISTRIBUTION IN THE UNITED KINGDOM, FRANCE, SPAIN AND GERMANY. - 2. MIXED COMPANIES IN THE ITALIAN LEGAL SYSTEM AND THE INTRICATE REGULATORY FRAMEWORK BEFORE THE DELEGATED LAW OF 7 AUGUST 2015, N. 124. - 3. THE REGULATORY INNOVATION: THE ART. 17 OF LEGISLATIVE DECREE 19 AUGUST 2016, N. 175, ON COMPANIES WITH MIXED PUBLIC-PRIVATE PARTICIPATION. 4. THE MIXED SOCIETY AS AN EXPRESSION OF PPPI AND THE PROMOTION OF THE EUROPEAN MODEL IN A CONTEXT OF LACK OF FINANCIAL AND ORGANIZATIONAL RESOURCES OF LOCAL AUTHORITIES. - 5. CONCLUSIONS OF THE RESEARCH. - 6. BIBLIOGRAPHY. 


\section{LE SOCIETÀ MISTE PER LA GESTIONE DI SERVIZI PUBBLICI LOCALI E LA LORO DIFFUSIONE NEL REGNO UNITO, IN FRANCIA, SPAGNA E GERMANIA}

Lo studio delle società di economia mista nelle diverse esperienze europee coinvolge l'attuale tendenza alla razionalizzazione della gestione dei servizi pubblici locali in conseguenza della crisi finanziaria ed economica; le difficoltà di bilancio e di risorse delle entità strumentali locali e di alcune forme societarie hanno, infatti, imposto nell'ordinamento italiano la revisione e il riordino del sistema delle società partecipate e della disciplina dei servizi pubblici locali, in attuazione delle deleghe di cui agli artt. 13-14 della legge 7 agosto 2015, n. 124, sulla riorganizzazione della pubblica amministrazione. A ciò si aggiunge un processo di trasformazione del governo locale, tendenza che riguarda diversi Paesi europei in cui il principio di autonomia è ormai in discussione e in via di "limitazione" soprattutto per esigenze di contenimento della spesa pubblica e di razionalizzazione delle modalità di gestione dei servizi pubblici locali. Le società a partecipazione pubblica sono ora disciplinate dal Decreto Legislativo 19 agosto 2016, n. 175, e i servizi pubblici locali di interesse economico generale erano oggetto di uno schema di decreto legislativo ${ }^{1}$ fino all'approvazione (24 novembre 2016) del Consiglio dei Ministri, ma l'atto normativo è stato ritirato a seguito della sentenza della Corte Costituzionale n. 251 del 2016 per una valutazione di opportunità ${ }^{2}$.

Alcuni ordinamenti giuridici europei (Regno Unito, Francia, Spagna, Germania) hanno da tempo applicato gli schemi giuridici della società di economia mista e della concessione per la gestione dei servizi pubblici, riconducibili all'ambito del partenariato pubblico privato istituzionalizzato,

1 V. Schema di decreto legislativo atto del Governo n. 308, dopo le successive integrazioni e modifiche a seguito del parere del Consiglio di Stato, Adunanza Commissione speciale, 6 aprile 2016, n. 1075 (in www.giustizia-amministrativa.it); il testo dello schema n. 308 e altri documenti sono reperibili in www.astrid-online.it, nelle sezioni "Concorrenza, mercato, regole", "Servizi pubblici locali".

2 La Corte Costituzionale, n. 451 del 25 novembre 2016, che ha dichiarato illegittime alcune disposizioni della legge delega n. 124/2015 nella parte in cui prevedono che il Governo adotti i decreti attuativi previo parere in sede di Conferenza unificata, anziché previa intesa in sede di Conferenza Stato-Regioni. Le disposizioni sono state ritenute illegittime in quanto incidenti su materie di competenza regionale, senza però individuare un ambito prevalente, con la conseguenza di assicurare il rispetto del principio di leale collaborazione che, nell'ambito del procedimento legislativo, viene garantito mediante il sistema delle Conferenze; non è sufficiente il parere come previsto dalla legge delega, ma è necessario un coinvolgimento più diretto delle autonomie regionali mediante l'intesa Stato/Regioni. Per la situazione di "stretto intreccio" fra competenze statali e regionali che caratterizza i settori coinvolti dalla delega, il principio di leale collaborazione assume rilievo come limite ulteriore che discende dalla Costituzione e che deve conformare l'esercizio del potere governativo nel senso che devono essere previste fasi che consentono forme adeguate di coinvolgimento delle Regioni. Per alcuni commenti v. A. Poggi, G. Boggero, Non si può riformare la p. a. senza intesa con gli enti territoriali: la Corte Costituzionale ancora una volta dinanzi ad un Titolo $V$ incompiuto, in www.federalismi.it, 28 dicembre 2016; R. BIfULCO, L'onda lunga della sentenza 251/2016 della Corte Costituzionale, in www.federalismi.it, 8 febbraio 2017. 
esaminato dall'UE in vari documenti ufficiali ${ }^{3}$ - atti di soft law - a partire dal 2004, ma senza elaborare una definizione normativa generale; peraltro, prima delle direttive del 2004 in materia di contratti pubblici, in diversi ordinamenti europei, il quadro delle regole applicabili nella scelta del partner privato era disomogeneo ${ }^{4}$, mentre si riscontrano maggiori similitudini negli anni successivi. Inoltre, mentre la disciplina del PPP è stata formulata in diversi Paesi europei in modo organico, quella del PPPI è ad oggi ancora carente, frammentaria o dispersa in diverse disposizioni sull'attività degli enti pubblici locali e sui contratti pubblici. Tuttavia, gli atti delle istituzioni europee hanno decisamente contribuito ad individuare un modello europeo di società mista che rientra nel PPPI.

La società di economia mista in Francia ${ }^{5}$ è disciplinata da una legge generale con disposizioni specifiche, mentre nel nostro ordinamento, fino al Decreto Legislativo n. 175/2016, risultava assai complicato ricostruire il quadro normativo: norme contenute nel Testo Unico degli Enti Locali (TUEL) e in diverse successive fonti legislative soprattutto di natura finanziaria e di razionalizzazione della spesa pubblica; solo di recente è emersa l'intenzione del legislatore per una risistemazione chiara della materia delle società partecipate e dei servizi pubblici locali. In Europa, la scelta di gestione mediante società miste è assai diffusa con riferimento a settori decisivi dell'economia; ciò può essere spiegato in una prospettiva di evoluzione storica: dalla prevalente gestione diretta dei servizi pubblici locali o mediante concessioni, soluzione ereditata dal secolo XIX secondo una logica di monopolio locale e nazionale, all'istituzione della Comunità europea che ha progressivamente inserito negli ordinamenti interni principi anche antitetici (concorrenza, libertà di prestazione, gestione secondo efficienza). Negli ultimi venticinque anni sono stati attuati rilevanti processi di privatizzazione in senso formale o sostanziale ${ }^{6}$ con diverse soluzioni e conseguenze circa la forma giudica privatistica, la proprietà del capitale e delle azioni, l'applicazione delle regole di concorrenza alle concessioni e alla fase di costituzione di società miste per la scelta del contraente privato.

\footnotetext{
${ }^{3}$ V. Commissione europea, Libro verde sulle partnership di tipo pubblico-privato e sul diritto comunitario degli appalti pubblici e delle concessioni (30 aprile 2004; COM (2004) 327 def.), in www.eur-lex.europa.eu.; Comunicazione interpretativa della Commissione 5 febbraio 2008, C (2007) 6661, in www.ec.europa.eu.

${ }^{4}$ Per le diverse soluzioni in alcuni Paesi europei (Francia, Germania, Spagna), si rinvia a D. DE PRETIS, Servizi pubblici locali e società miste: una visione comparativa, in M. P. CHITI (a cura di), Le forme di gestione dei servizi pubblici locali tra diritto europeo e diritto locale, il Mulino, Bologna, 2006, 104 ss.

${ }^{5}$ Per il caso dell'ordinamento francese, si rinvia a S. Antoniazzi, Società miste e servizi pubblici locali. Esperienze nazionali e modello europeo, Editoriale Scientifica, Napoli, 2017, 62 ss.

${ }^{6}$ Per un'analisi economica e statistica sui risultati della privatizzazione in senso formale e materiale nelle diverse esperienze dei Paesi europei, si rinvia a C. SCHMITT, The diffusion of privatisation in Europe: political affinity or economic competition?, in Public Administration, 2014, 615 ss. Per il caso particolare del servizio idrico nel Regno Unito v. S. ANTONIAZZI, La privatizzazione del servizio idrico in Inghilterra e in Galles e la riforma Water Act 2014, in M. MidiRI, S. AntoniAzzi (a cura di), Servizi pubblici locali e regolazione, Editoriale Scientifica, Napoli, 2015, 255 ss.
} 
Il modello societario di economia mista comporta, come emerge dai noti atti europei ${ }^{7}$ sul partenariato pubblico privato istituzionalizzato (PPPI), un'entità giuridica separata di appartenenza pubblico/privata con il compito principale di assicurare il reperimento di finanziamenti e l'erogazione di un pubblico servizio oppure la realizzazione di un progetto di infrastruttura nell'interesse pubblico. La collaborazione diretta tra pubblico e privato garantisce comunque alla parte pubblica mediante la presenza nell'azionariato e negli organi decisionali, di conservare un elevato grado di controllo dell'attuazione del progetto; inoltre, l'entità societaria consente anche al partner pubblico di sviluppare e migliorare la propria esperienza nella gestione dei servizi pubblici con l'aiuto della parte privata. La società mista può derivare dall'istituzione di un'entità controllata dal privato e dal pubblico o dal controllo privato assunto in un'impresa pubblica oppure dalla partecipazione di un partner privato in una società pubblica preesistente sulla base di un contratto pubblico o di una concessione.

L'attribuzione a privati di funzioni pubbliche nella forma del partenariato pubblico privato (PPP), nonostante la prevalente privatizzazione dei servizi pubblici, ha origine ed è molto diffusa nel Regno Unito $^{8}$, mentre in Germania ${ }^{9}$ si è sempre affermata una tradizionale resistenza ad affidamenti con gara a privati in concessione o mediante società mista, prevalendo a lungo la logica della gestione diretta con affidamenti "fiduciari" a privati. La scelta del partner privato di società a partecipazione pubblica non era sottoposta alle regole della gara, ritenendo la disciplina degli appalti pubblici non applicabile ai contratti associativi; di certo sono derivate innovazioni dal recepimento delle direttive UE sui contratti pubblici del 2004 e, più recentemente, dalla trasposizione ${ }^{10}$ delle direttive del 2014.

In una posizione intermedia, invece, in Francia e in Spagna ${ }^{11}$ : vi sono significative differenze, ma è evidente la resistenza alla soluzione della concorrenza nel settore dei servizi pubblici; in Francia si era soprattutto diffusa la gestione locale delegata nella forma di affidamenti mediante una trattativa privata, mentre era prevista la gara per gli affidamenti di servizi pubblici e quelli inerenti alla realizzazione di operazioni di aménagement.

\footnotetext{
${ }^{7}$ Si rinvia a nota 3.

${ }^{8}$ V. S. ANTONIAZZI, Società miste e servizi pubblici locali, cit., 45 ss. V. altresì M. Dugato, Il partenariato pubblico-privato: origine dell'istituto e sua evoluzione, in $\mathrm{F}$. MASTRAgOSTino (a cura di), La collaborazione pubblico-privato e l'ordinamento amministrativo, Giappichelli, Torino, 2011, 55 ss.

${ }^{9}$ S. ANTONIAZZI, op. ult. cit., 120 ss.

10 Per gli appalti pubblici la disciplina Gesetz zur Modernisierung des Vergaberechts (Vergaberechtsmodernisierungsgesetz VergRModG) del 17 febbraio 2016 (in Bundesgesetzblatt Jahrgang Teil I Nr. 8, ausgegeben zu Bonn am 23 Februar 2016, 203) e Verordnung zur Modernisierung des Vergaberechts (VergRModVO), del 12 aprile 2016 (in Bundesgesetzblatt Jahrgang Teil I Nr. 16, ausgegeben zu Bonn am 14 April 2016, 624). Per le concessioni v. Konzessionsvergabeverordnung (KonzVgV) del 12 aprile 2016 (in Bundesgesetzblatt Jahrgang Teil I Nr. 16, ausgegeben zu Bonn am 14 April 2016, 683).

${ }^{11}$ S. AntoniAzZi, op. ult. cit., 93 ss.
} 
Nell'ordinamento spagnolo, già prima della trasposizione delle direttive UE n. 23 e n. 24 del 2014 mediante la Ley 9/2017 dell'8 novembre 2017, sui contratti del settore pubblico, e delle precedenti direttive del 2004, gli enti di diritto pubblico erano considerati enti aggiudicatori ed erano previste gare pubbliche per l'istituzione di società di economia mista di gestione di servizi pubblici e, infatti, nella legge sui contratti dello Stato (1965) questo schema era considerato una forma di contratto amministrativo; così nell'ambito dell'amministrazione locale, la società mista è stata considerata una modalità del contratto amministrativo e, quindi, con selezione del partner privato mediante procedura pubblica (legge sui contratti delle amministrazioni pubbliche del 1995).

\section{LE SOCIETÀ MISTE NELL'ORDINAMENTO ITALIANO E L'INTRICATO QUADRO NORMATIVO PRIMA DELLA LEGGE DELEGA 7 AGOSTO 2015, N. 124}

Per tradizione storica i servizi pubblici locali ${ }^{12}$ sono stati prevalentemente gestiti da enti locali o mediante concessioni in un contesto di monopolio (locale e nazionale), fino all'istituzione della Comunità Europea che ha, invece, affermato una logica del tutto diversa secondo concorrenza, libertà di prestazione e di gestione efficiente dei servizi pubblici; gli Stati membri hanno, quindi, modificato progressivamente la politica di gestione costretti da esigenze di risorse e di efficienza adottando soluzioni di privatizzazione e di concorrenza, sotto I'influenza dell'UE per le concessioni e la costituzione di società miste. La scelta per un'effettiva concorrenza tra privati appare più accentuata nel Regno Unito sulla base di un risalente deciso indirizzo di politica dei servizi pubblici confermato nel tempo, mentre i casi di ripubblicizzazione sono dipesi da fallimenti della gestione privata o dell'istituito PPP (ad es., per la London Tube nel Regno Unito ${ }^{13}$ ). Ben diversa la soluzione prevalente nell'ordinamento tedesco, in cui per tradizione risalente la gestione dei

\footnotetext{
${ }^{12}$ Si rinvia a F. MeRuSI, voce Servizio pubblico, in Nuoviss. Dig. Ital., XVII, 1970, 215 ss., ancora attuale per scoprire l'origine del service public nella giurisprudenza francese che distingueva tra actes d'autorité e actes de gestion della pubblica amministrazione e per quest'ultima categoria vi era "una zona grigia" (contratti di lavori pubblici e di forniture, alcune concessioni e altre obbligazioni) con un regime speciale di privilegio rispetto alla disciplina di diritto privato) in seguito attribuita al giudice amministrativo (inizi del secolo scorso); il service public assumeva il ruolo di criterio generale sulla competenza del Consiglio di Stato per atti non autoritativi sottoposti a regole non incluse nel diritto comune. In Italia, invece, il dibattito era incentrato sulla municipalizzazione dei servizi pubblici e, quindi, sull'attribuzione agli enti locali (Comuni, Province) di attività economiche destinate prevalentemente alle comunità locali; nell'ordinamento costituzionale, ai sensi dell'art. 43 Cost. e dell'art. 1 della legge sulla municipalizzazione "il servizio pubblico è una clausola generale che si riferisce ad un fenomeno ai cui indici di esistenza saranno individuati, a seconda dei casi, dal legislatore o dall'ente locale territoriale e in fine riesaminati, sotto il profilo della $<<$ ragionevolezza $>>$, dal giudice costituzionale o dal giudice amministrativo". Sulla legittimità della partecipazione del Comune in una società per azioni nella risalente disciplina v. M. MAZZARELLI, La società per azioni con partecipazione comunale, Giuffrè, Milano, 1987, 23 ss.

${ }^{13}$ V. S. ANTONIAZZI, op. ult. cit., 53 ss.
} 
servizi pubblici è competenza di enti statali o locali, dato che emerge un ritorno alla gestione pubblica dopo una fase di privatizzazione, soprattutto in conseguenza di referendum, strumento che ha consentito ai cittadini di modificare la politica dei servizi pubblici, considerati gli elevati costi imposti dalla gestione di società private e miste.

Soluzioni intermedie sono presenti in Francia e Spagna in cui attualmente vi sono significative restrizioni per esigenze di finanza pubblica; in Francia prevale ancora la gestione delegata dei servizi pubblici locali sulla base di affidamenti in concessione e nuove forme societarie, ma con diversi casi di ripubblicizzazione ${ }^{14}$. In Spagna la disciplina sulle società miste ${ }^{15}$ e pubbliche è stata modificata in varie fasi in conseguenza delle direttive del 2004 e delle recenti riforme circa le autonomie territoriali e la razionalizzazione della spesa pubblica; ulteriori innovazioni sono derivate dalla trasposizione delle direttive (UE) n. 23 e n. 24 del 2014 in materia di contratti pubblici mediante la Ley 9/2017 che, circa l'aggiudicazione di contratti di concessione di servizi a società miste, nella Disposición adicional vigésima segunda ha stabilito per il capitale misto la necessaria partecipazione pubblica maggioritaria.

L'orientamento di ritorno alla gestione pubblica dipende prevalentemente dalla recente alternanza politica nelle amministrazioni locali e da movimenti d'opinione dei cittadini, ma è di difficile attuazione.

In generale, le ragioni che inducono a ritornare alla gestione pubblica attengono ai costi e all'efficienza in relazione a servizi specifici, motivi che hanno anche giustificato la privatizzazione; più raramente prevalgono motivazioni ideologiche. Si è, quindi, diffusa in Europa una tendenza generale di riforma del settore per obiettivi di contenimento della spesa pubblica e di qualità delle prestazioni nonché in conseguenza del recepimento delle direttive del 2014.

In Italia non emerge una significativa tendenza a rimunicipalizzare ${ }^{16} \mathrm{i}$ servizi attualmente gestiti da società miste o private, tranne in casi di fallimento di queste modalità di gestione; vi sono alcuni orientamenti politici favorevoli e per il servizio idrico con il referendum del 2011 è emersa una chiara posizione contraria alla privatizzazione e alla liberalizzazione, dato che gli elettori si sono pronunciati per l'abrogazione dell'art. 23bis del Decreto Legge 25 giugno 2008, n. 112 (convertito nella

\footnotetext{
${ }^{14}$ S. ANTONIAZZI, op. ult. cit., 73 ss.

15 Per approfondimenti, D. SANTIAGo IgLESIAS, Las sociedades de economía mixta come forma de gestión de los servicios públicos locales, Madrid, Iustel, 2010; ID., Las sociedades municipales, in M. Almeida Cerreda, C. Tubertini, P. Costa Gonçalves (dirs.), D. SANTiAgo Iglesias, F. Di LAScio, P. CRUz Silva (coords.), La racionalización de la organización administrativa local: las experiencias española, italiana y portuguesa, Civitas, Thomson Reuters, Navarra, 2015, 230 ss.; ID., Empresas locales, in F. VELASCO CABAllero (dir.), Tratado de derecho económico local, Marcial Pons, Madrid, 2017, 171 SS.

${ }^{16}$ Per uno studio della recente evoluzione, v. G. PIPERATA, Tendencias recientes en las reformas de la normativa italiana sobre los servicios públicos locales: de la remunicipalización hacia la desmunicipalización, in T. FONT I LLOVET, J. J. DíEz SÁNCHEZ (Coords.), Los servicios públicos locales. Remunicipalización y nivel óptimo de gestión, Iustel, Madrid, 2017, 221 ss.
} 
legge 6 agosto 2008, n. 133) che prevedeva una decisa limitazione delle gestioni pubbliche e favoriva un'apertura obbligatoria agli operatori privati; in seguito, vi sono state difficoltà rilevanti di adeguamento normativo anche per le sentenze della Corte Costituzionale, oltre ad un acceso dibattito ${ }^{17}$.

Negli ultimi dieci anni, per esigenze di riduzione della spesa pubblica e di attuazione del principio di concorrenza, la questione della razionalizzazione $^{18}$ delle società partecipate degli enti locali ${ }^{19}$ e delle relative partecipazioni ha assunto una rilevanza centrale in tutte le politiche di riforma delineate dai vari Governi, pur non sempre efficaci e coerenti nella loro evoluzione; infatti, con interventi normativi diversi ${ }^{20}$ si è tentato di limitare l'attività imprenditoriale degli enti locali, soprattutto nella gestione dei servizi pubblici, prevedendo limiti per la costituzione di nuove società, per l'azione in quelle esistenti oppure mediante divieti ad acquisire partecipazioni in società già attive nel mercato e da ciò è emersa una tendenza decisivamente opposta al significativo utilizzo di strumenti privatistici negli anni precedenti, soprattutto considerati i risultati non particolarmente efficienti emersi nel periodo di crisi finanziaria ed economica ${ }^{21}$. Peraltro, i tentativi di razionalizzazione mediante i frequenti interventi normativi non sono stati efficaci, dato che comunque dal 2008 le società partecipate sono, invece, aumentate nonostante l'incremento

17 Sulla complessa questione v. G. PIPERATA, Liberalizzazioni e interesse generale: il caso del servizio idrico integrato, in Diritti Lavori Mercati, 2015, II, 6 ss.

${ }_{18}$ Si rinvia a A. MARRA, La razionalizzazione delle società partecipate dagli enti locali dopo la legge di stabilità 2015, in Dir. econ., 2015, 299 ss.

${ }^{19}$ V. M. DugATo, Le società a partecipazione pubblica, in Giorn. dir. amm., 2013, 855 ss.

20 Si rinvia all'art. 13 del Decreto Legge n. 223 del 2006, che ha introdotto I'obbligo per le società strumentali di cessare le attività non consentite entro un certo termine e un meccanismo di nullità dei contratti già stipulati in violazione di norme ivi stabilite; I'art. 3, comma 27, della legge finanziaria 2008 (legge n. 244 del 2007) che ha previsto un divieto per tutte le amministrazioni di costituire nuove società o di acquisire o conservare partecipazioni in società preesistenti per attività non strettamente necessarie per I'esecuzione delle finalità istituzionali. L'art. 14, comma 32, del Decreto Legge n. 78 del 2010, ha previsto limitazioni per la costituzione e la conservazione di partecipazioni in società. In seguito, sono intervenute ulteriori disposizioni: l'art. 4 del Decreto Legge $n$. 95 del 2012, che ha introdotto l'obbligo di scioglimento o l'alienazione delle partecipazioni entro una certa data per le società strumentali il cui fatturato per le prestazioni di servizi a favore di amministrazioni superiore al 90\% dell'intero fatturato; l'art. 1, comma 568bis, della legge n. 147 del 2013 che ha previsto agevolazioni fiscali per quelle amministrazioni locali e società controllate in caso di scioglimento di queste o di alienazione delle partecipazioni detenute. Altri atti normativi hanno introdotto alcune norme relative al settore anche se non direttamente destinati alla razionalizzazione, così l'art. 23bis del Decreto Legge n. 112 del 2008 (decreto Tremonti) circa le forme di gestione dei servizi pubblici locali e l'eccezionalità dell'affidamento in house, e l'art. 4 del Decreto Legge $n$. 138 del 2011 che ha riprodotto l'art. 23bis, che in realtà era stato abrogato in conseguenza del referendum popolare e poi dichiarato, di conseguenza, incostituzionale (sentenza n. 199 del 2012).

${ }^{21}$ Per una certa contraddizione negli orientamenti v. S. MAROTTA, La spending review nei servizi pubblici locali: necessità di razionalizzare, volontà di privatizzare, in Munus, 2014, 261 ss.; cfr., R. URSI, Il governo del gruppo pubblico locale al tempo della Spending review, in M. PASSALACQUA (a cura di), Il < <disordine>> dei servizi pubblici locali, Giappichelli, Torino, 2015, 145 ss. 
dei vincoli di finanza pubblica per gli enti locali ${ }^{22}$; del resto, è riscontrabile una certa tradizione, risalente agli anni '90 del secolo scorso, di costituzione di società miste finalizzate al coinvolgimento di altri soggetti, pubblici e privati, per il finanziamento di servizi pubblici e la gestione imprenditoriale mediante società per azioni come "fuga" dalla municipalizzazione ${ }^{23}$. A questo contesto già complesso si aggiungono difficoltà per le competenze come previste dal dall'art. 3bis del Decreto Legge n. 138/2011, che sembra aver moltiplicato gli enti governo per la gestione dei servizi pubblici locali e prodotto una significativa frammentazione, ulteriormente complicata dalla legge c.d. Delrio ${ }^{24}$ per funzioni attribuite alla città metropolitana e la soppressione di enti a cui prima spettavano competenze di organizzazione per ambiti provinciali (art. 1 della legge n. 56/2014, commi 44 e 90).

L'intenzione recente del legislatore (legge delega n. 124/2015) è quella di razionalizzare le società partecipate e quelle in house assai diffuse (e diverse in situazione di dissesto finanziario) e di conservare, ma con intensi controlli e limitazioni di natura organizzativa, finanziaria e di

${ }^{22}$ V. A. MARRA, La razionalizzazione delle società partecipate degli enti locali dopo la legge di stabilità 2015, cit., 300. Per la rilevante diffusione di società a partecipazione pubblica, si rinvia alla ricerca di D. MARCHESI, Riordino delle partecipate degli enti locali, criticità, possibili policy, interventi in atto, in Analisi giuridica dell'economia, 2015, 335 ss.

23 Si rinvia a F. Merusi, Servizi pubblici instabili, il Mulino, Bologna, 1990, 37 ss., spec. 42-45; secondo l'Autore l'affidamento in concessione di servizi pubblici a privati è finalizzato all'ammortamento dei costi di costruzione di opere pubbliche necessarie per la gestione dei servizi da parte del privato concessionario ed è una soluzione adottata anche all'inizio del XX secolo. Vi sono però due novità: la concessione può essere attribuita anche ad una società per azioni a cui partecipa il comune concedente, che può in tal modo controllare direttamente l'operazione, e il leasing che consente il finanziamento dell'opera senza ricorrere alla finanza pubblica. Per alcuni studi di riferimento, cfr., M. CAMmELli, I servizi pubblici locali dell'amministrazione locale, in Le Regioni, 1992, 24 ss.; M. CAMmelLI, A. ZiRoldi, Le società s partecipazione pubblica nel sistema locale, Maggioli, Rimini, 1997; P. ABBADESSA, Le società miste per i servizi locali: profili organizzativi speciali, in F. TRIMARCHI (a cura di), Le società miste per i servizi locali, Giuffrè, Milano, 1999, 87 ss.; D. SORACE, Pubblico e privato nella gestione dei servizi pubblici locali mediante società per azioni, ivi, 135 ss.; M. DUGATO, Le società per la gestione nei servizi pubblici locali, Il Giornale di diritto amministrativo, Quaderni, Ipsoa, Milano, 2001; M. DugATo, Il servizio pubblico locale: incertezze qualificatorie e tipicità delle forme di gestione, in Giorn. dir. amm., 2002, 24 ss.; M. CAMmELLI, Concorrenza, mercato e servizi pubblici: le due riforme, in Riv. trim. app., 2003, 517 ss.; M. Dugato, Le società degli enti territoriali alla luce dell'art. 13 del D. L. n. 223/2006, in M. CAMmeLLI, M. Dugato (a cura di), Studi in tema di società a partecipazione pubblica, Giappichelli, Torino, 2008, 347 ss. Per un'indagine sulle società per la gestione dei servizi pubblici locali e sui controlli, v. G. MoRBIDELLI, I controlli sulle società a partecipazione pubblica, in G. Morbidelli, Scritti di diritto dell'economia, Giappichelli, Torino, 2001, 279 ss., e in A. PREDIERI (a cura di), Controlli societari e governo dell'impresa, Giappichelli, Torino, 1999, 99 ss.

${ }^{24}$ Per un'analisi critica del nuovo ordinamento amministrativo regionale e locale, si rinvia a C. TUBERTINI, L'attuazione regionale della legge 56/2014: verso un nuovo assetto delle funzioni amministrative, in Le Regioni, 2016, 99 ss. Per uno studio approfondito del riordino dell'amministrazione locale in Italia e di diverse esperienze europee $v$. L. VANDELli, G. GARDini, C. TUBERTINi (a cura di), Le autonomie territoriali: trasformazioni e innovazioni dopo la crisi, Maggioli, Rimini, 2017. 
rendicontazione, le gestioni pubbliche già esistenti e l'attuale modello di società mista con capitale parzialmente pubblico ${ }^{25}$ nella forma di struttura "chiusa" non quotata nei mercati regolamentati e di società per azioni c.d. multiservizi ${ }^{26}$ quotata, imprese che gestiscono i servizi pubblici di molti Comuni. In quest'ultimo schema sono incluse entità societarie (anche nella forma di società holding capogruppo con compiti di direzione e di coordinamento) che per l'estensione dell'attività sono di "dimensione regionale", radicate nel territorio per la presenza degli azionisti enti locali e con estensioni nelle Regioni limitrofe, in conseguenza di aggregazioni, sollecitate anche dalla legge ${ }^{27}$, e per l'incorporazione di medie imprese; nell'azionariato vi è una presenza significativa di azionisti privati che controllano, anche se limitatamente, l'azionariato pubblico ${ }^{28}$.

Nel panorama sono anche presenti aziende private, ma in numero decisamente minore; in Italia, infatti, per tradizione giuridica, gli enti locali possono decidere discrezionalmente la modalità di gestione di un servizio pubblico locale di rilevanza economica ed affidarlo a una società in house

${ }^{25}$ Per i caratteri fondamentali della società mista per l'esternalizzazione del servizio: G. PIPERATA, Le società a partecipazione pubblica nella gestione dei servizi degli enti locali, in M. CAMmelli, M. Dugato (a cura di), Studi in tema di società a partecipazione pubblica, cit., 312 ss.; R. URSI, Società ad evidenza pubblica, Editoriale Scientifica, Napoli, 2012, ristampa 2013. Per un recente studio, v. D. ANSELMI, L'affidamento a società miste, in A. Vigneri, M. Sebastiani (a cura di), Società pubbliche e servizi locali, Astrid, 2016, in www.astrid-online.it. Per un'approfondita analisi delle società miste nella disciplina fino al 1995: G. MoRBIDELLI, Società miste, servizi pubblici e opere accessorie, in Riv. trim. appalti, 1997, 493 ss.; mentre esprime una tendenza in senso riduttivo dell'utilizzo delle società miste in conseguenza degli interventi (fino al 2006) della Corte di Giustizia in materia di in house providing e da ciò "la progressiva erosione dell'autonomia organizzativa degli Stati membri": M. P. CHITI, Verso la fine del modello di gestione dei servizi pubblici locali tramite società miste, in Foro amm. TAR, 2006, II, 1161 ss. Più recentemente, sulle ragioni della costituzione della società mista che non deriva tanto dalla ricerca di un'effettiva partnership, ma dall'esigenza di garantire l'ammortamento dei beni e degli investimenti, v. M. DUGATO, Le società a partecipazione mista per la gestione dei servizi pubblici locali. Il procedimento di costituzione, l'affidamento dei lavori e la relazione tra ente socio e società, in M. DugATo, F. MASTRAgostino (a cura di), Partecipazioni, beni e servizi pubblici tra dismissioni e gestione, Bononia University Press, Bologna, 2014, 213 ss., spec. 227-228; per questa interpretazione anche F. MERUSI, v. nota 23.

${ }^{26} \mathrm{~V}$. ad es., le società $A 2 A$ spa (sede legale a Brescia, www.a2a.eu); Acea spa (sede legale a Roma, www.acea.it); Hera spa (sede legale a Bologna, www.gruppohera.it; nel Gruppo Hera anche Acegasapsamga spa, sede a Trieste, www.acegasapsamga.it); Iren spa (sede legale a Reggio Emilia, www.gruppoiren.it). Per uno studio analitico e riferimenti alla pianificazione industriale, si rinvia a S. VENIER, Le prospettive strategiche e gestionali dei servizi pubblici locali in Italia, in M. MidirI, S. ANTONIAZZI (a cura di), Servizi pubblici locali e regolazione, cit., 80 ss.

${ }^{27}$ Il Decreto Legge 12 settembre 2014, n. 133 (c.d. decreto "Sblocca Italia"), convertito nella legge 11 novembre 2014, n. 164, ha previsto un rilancio delle privatizzazioni per le società partecipate dagli enti locali, in particolare nei settori del trasporto locale, servizi ambientali (rifiuti) e dell'idrico.

28 Ad es., nel caso della Società Hera spa, l'azionariato al 31 marzo 2018 è così composto: 49,5\% Patto soci pubblici e 50,5\% flottante (azioni circolanti che gli investitori possono commerciare nel mercato), si rinvia al sito www.gruppohera.it. Per una ricostruzione storica dell'attività di Hera spa: C. DALL'Osso, Una società verso il futuro, I servizi pubblici locali nella prospettiva di Hera, La Mandragora, Imola, 2009. 
o a una società mista partecipata dall'amministrazione affidante (o da più amministrazioni) e da privati oppure ad un'impresa privata individuata con gara. Le società miste, come è noto, sono riconducibili al partenariato pubblico privato istituzionalizzato ${ }^{29}$ e l'affidamento deriva da una gara che ha ad oggetto la selezione del socio privato e la concessione del servizio, secondo un meccanismo di gara "a doppio oggetto"30; negli ultimi anni il modello ha attirato maggiori attenzioni per esigenze di qualità dei servizi erogati e per la necessità pressante di reperire nuove risorse a fronte dei vincoli stringenti imposti dalla finanza pubblica. Inoltre, la società mista può derivare dalla trasformazione di società pubbliche a seguito di parziale privatizzazione ${ }^{31}$.

In effetti, lo strumento della società mista potrebbe limitare gli svantaggi dipendenti dalla gestione privata e da quella pubblica e consentire l'attuazione di finalità pubbliche di lungo periodo unitamente allo scopo lucrativo che consente di reperire risorse. Rispetto alla gestione privatistica, la rinegoziazione delle clausole contrattuali potrebbe essere più agevole e risolta dall'organo di gestione, mentre potrebbero essere facilitati e rafforzati il controllo dell'adempimento da parte dell'ente locale socio pubblico e l'estensione di vincoli pubblicistici al socio privato ${ }^{32}$; inoltre, rispetto ad una gestione esclusivamente pubblicistica, la presenza del partner privato potrebbe garantire nuovi investimenti. Ulteriori vantaggi potrebbero derivare dall'attribuzione al socio privato di una posizione significativa nella gestione mediante una partecipazione maggioritaria e il socio pubblico potrebbe esercitare poteri minimi per intervenire nella gestione ed esercitare i controlli per la tutela di interessi pubblici ${ }^{33}$.

Quindi, nell'ordinamento italiano coesistono diverse soluzioni di gestione con alcune contraddizioni che derivano dalla risalente privatizzazione formale da cui sembra sia conseguita in realtà un'espansione della pubblicizzazione sostanziale ${ }^{34}$ e, di recente, un

\footnotetext{
${ }^{29}$ V. Comunicazione 5 febbraio 2008, C (2007) 6661, della Commissione europea, in www.ec.europa.eu.

${ }^{30}$ Circa la scelta del socio privato delle società miste, I'obbligatorietà della gara pubblica e l'eventuale elusione delle regole mediante la circolazione delle partecipazioni e I'aumento del capitale sociale v. S. VALAGUZZA, Società miste a partecipazione comunale, Giuffrè, Milano, 2012, 159 ss.; spec. 166-167.

31 Per questa soluzione v. G. PIPERATA, La trasformazione delle società a partecipazione pubblica. Dalla società in house alla società a partecipazione mista, in M. Dugato, F. MAStRAgostino (a cura di), Partecipazioni, beni e servizi pubblici tra dismissioni e gestione, cit., 151 ss. Più in generale sulle modalità di gestione, v. G. PIPERATA, Tipicità e autonomia dei servizi pubblici locali, Giuffrè, Milano, 2005.

32 Sul ruolo del socio pubblico e sul regime di circolazione delle partecipazioni sociali nelle società miste e l'estensione dei vincoli pubblicistici, v. G. M. CARUSO, Il socio pubblico, Jovene, Napoli, 2016, 149 ss. e 216 ss.

33 Per questa soluzione di prevalenza della partecipazione privata, v. R. OcchILUPo, G. RomA, Le società miste affidatarie di servizi pubblici locali: un'analisi degli assetti di governo, in Analisi giuridica dell'economia, 2015, 496.

34 In tal senso, F. Merusi, Il sogno di Diocleziano. Il diritto delle crisi economiche, Giappichelli, Torino, 2013, 90-91; secondo I'Autore "Le aziende organo municipalizzate sono state trasformate in società per azioni, e fin qui sembrerebbe trattarsi del solito
} 
incremento dei vincoli di natura pubblicistica come conseguenza della rilevanza del debito pubblico, delle limitate risorse a disposizione e della crisi finanziaria ed economica; per questi aspetti il percorso della recente evoluzione è molto simile a quello dell'ordinamento spagnolo.

\section{L'innovazione normativa: I'art. 17 del Decreto Legislativo 19 agosto 2016, n. 175, sulle società a partecipazione mista pubblico- privata}

Il nuovo contesto normativo è ancora assai complesso, ma con norme forse più coordinate e coerenti rispetto al passato; occorre, infatti, considerare il Decreto Legislativo n. 50/2016 sugli appalti e contratti pubblici $^{35}$ e l'attuazione del Decreto Legislativo 19 agosto 2016, n. 175, sulle società a partecipazione pubblica ${ }^{36}$ con norme assai puntuali che confermano un intenso controllo sulla razionalizzazione delle società partecipate e sulla scelta dell'ente locale, disposizioni di diritto speciale nonché limitazioni dell'iniziativa economica privata; per la razionalizzazione periodica, si aggiunge il ruolo assai significativo della Corte dei Conti (art. 20), che deve verificare i relativi piani sottoposti dagli enti locali con un'apposita relazione tecnica ${ }^{37}$.

Infatti, il Testo Unico in materia di società partecipate stabilisce in senso assolutamente restrittivo, superando ogni dubbio, che "Le

fenomeno della privatizzazione "formale", sennonché, per giustificare l'affidamento di un servizio pubblico a tali società apparentemente private, è stata inventata dalla giurisprudenza comunitaria, per legittimare il fenomeno, non esclusivamente italiano, dal punto di vista dell'applicabilità della normativa comunitaria, la figura della società in house, cioè della società per azioni che, nella sostanza, corrisponde ad un organo dell'ente locale, come era, anche nella forma, la vecchia azienda municipalizzata. Ma non basta. Buona parte degli enti locali non si sono limitati a privatizzare formalmente le aziende municipalizzate facendone un organo di diritto privato, ma, vista l'utilità della società per azioni sotto vari profili (soprattutto in funzione elusiva di norme di diritto pubblico), ha provveduto ad affidare a società per azioni servizi di ogni genere".

${ }^{35} \mathrm{~V}$. Decreto Legislativo 18 aprile 2016, n. 50, di recepimento delle Direttive n. 23, n. 24 e n. 25 del 2014, integrato dal Decreto Legislativo correttivo 19 aprile 2017, n. 56.

36 Per approfondimenti, si rinvia a S. ANTONIAZZI, op. ult. cit., 215 ss.; v. altresì S. SABLONE, Art. 17. Società a partecipazione mista pubblico-privata, in G. Meo, A. Nuzzo (diretto da), Il Testo Unico sulle società pubbliche, Cacucci, Bari, 2016, 249 ss.; sui profili pubblicistici e societari delle società miste, V. DoNATIVI, Le società a partecipazione pubblica, Wolters Kluwer, Assago, 2016, 1082 ss.; circa i processi decisionali e gestionali delle società a partecipazione pubblica per la soddisfazione degli interessi pubblici $v$. A. ZIRUOLo, Valore pubblico e società partecipate. Tendenze evolutive della performance, Franco Angeli, Milano, 2016.

37 Già è vigente il controllo dei piani di razionalizzazione delle società partecipate predisposti dagli enti pubblici da parte della Corte dei Conti, come previsto art. 1, commi 611 e 612 della legge 23 dicembre 2014, n. 190) e, in particolare, si richiama il referto della Sezione regionale di controllo per I'Emilia-Romagna (deliberazione dell'Adunanza $\mathrm{n}$. 32/2016/VSGO, 24 marzo 2016); ad es., circa le società quotate, la Corte dei Conti rileva che diversi enti locali non hanno considerato la partecipazione in Hera spa (v. § 4.1.1.) "in quanto società quotata nei mercati regolamentati e pertanto disciplinata da specifica normativa di settore", ma è stata eccepita la non adeguatezza della motivazione "considerata comunque I'alienabilità, almeno potenziale, della partecipazione e tenuto conto che numerosi degli altri enti locali partecipanti hanno incluso la partecipazione stessa nel proprio piano di razionalizzazione". 
amministrazioni pubbliche non possono direttamente o indirettamente, costituire società aventi per oggetto attività di produzione di beni e servizi non strettamente necessarie per il perseguimento delle proprie finalità istituzionali, né acquisire o mantenere partecipazioni, anche di minoranza, in tali società" (art. 4, comma 1); sono definite le finalità perseguibili mediante l'acquisizione e la gestione di partecipazioni pubbliche, quali, ad es., la produzione di un servizio di interesse generale, la progettazione e realizzazione di un'opera pubblica e anche "c) realizzazione e gestione di un'opera pubblica ovvero organizzazione e gestione di un servizio d'interesse generale attraverso un contratto di partenariato di cui all'articolo 180 del Decreto Legislativo n. 50 del 2016, con un imprenditore selezionato con le modalità di cui all'art. 17 , commi 1 e 2 ".

L'art. 17, comma 1 , sulle società a partecipazione mista pubblicoprivata, stabilisce la quota di partecipazione del soggetto privato che non può essere inferiore al trenta per cento - con possibilità di diverse combinazioni e di una partecipazione pubblica minoritaria - e la procedura di selezione pubblica del medesimo, nel rispetto dell'art. 5, comma 9, del Decreto Legislativo n. 50 del 2016, e "ha ad oggetto, al contempo la sottoscrizione o l'acquisto della partecipazione societaria da parte del socio privato e l'affidamento del contratto di appalto o di concessione oggetto esclusivo dell'attività della società mista" quindi, mediante una gara a doppio oggetto ${ }^{38}$. Inoltre, i commi 2-5 dell'art. 17, prevedono puntuali vincoli normativi, oltre al possesso da parte del socio privato dei requisiti di qualificazione contenuti in norme legali o regolamentari in relazione alla prestazione; già all'avviso pubblico devono essere allegati la bozza dello statuto e degli accordi parasociali, degli elementi essenziali del contratto di servizio e dei regolamenti di esecuzione. Il bando di gara deve precisare l'oggetto dell'affidamento, i requisiti di qualificazione (generali, speciali, tecnico ed economico-finanziari) dei partecipanti e ovviamente un criterio di aggiudicazione che assicuri la concorrenza ${ }^{39}$; si aggiungono limiti riguardo alla durata della partecipazione privata alla società, che non può essere superiore alla durata dell'appalto e della concessione per l'affidamento e l'esecuzione, e per il contenuto dello statuto (circa meccanismi idonei per lo scioglimento della società nei casi di risoluzione del contratto di servizio, di trasformazione, fusione o mutamento della titolarità del controllo sul soggetto privato partecipante, di cessione o affitto da parte di questo dell'azienda o del ramo d'azienda coinvolto nell'esecuzione dell'appalto o della concessione).

\footnotetext{
${ }^{38}$ V. art. 23-bis, comma 2, lett. b) del Decreto Legge 25 giugno 2008, n. 112, e s.m.; cfr., parere del Consiglio di Stato, Sez. II, 18 aprile 2007, n. 456, in www.giustiziaamministrativa.it; Corte di Giustizia, Sez. III, 15 ottobre 2009, C-196/09, in www.curia.europa.eu.

39 Per approfondimenti, G. PIPERATA, La partecipazione delle pubbliche amministrazioni a società di capitali nel nuovo disegno di riforma: modelli, procedure e limiti, in F. CERIONI (a cura di), Le società pubbliche nel Testo Unico, Giuffrè, Milano, 2017, 1 ss.
} 
Circa il riferimento ordinamentale per le società a partecipazione pubblica $^{40}$, è previsto il rinvio generale alle "norme sulle società contenute nel codice civile e in leggi speciali" (art. 1, comma 3, del T. U.). Tuttavia, sono precisate deroghe a norme al codice civile per il controllo interno del socio pubblico sulla gestione dell'impresa (artt. 2380-bis e 2409-novies c. c.) disciplinato da clausole degli statuti delle società per azioni; vi sono deroghe all'art. 2479, comma 1, c.c. per l'attribuzione di particolari diritti (art. 2468, comma 3, c.c.) che possono essere inseriti negli statuti delle società a responsabilità limitata, e all'art. 2479, comma 1, c.c. per eliminare o limitare la competenza dei soci. Per le società per azioni è, inoltre, consentita l'emissione di speciali categorie di azioni anche con prestazioni accessorie da assegnare al socio privato; i patti parasociali possono avere una durata superiore a cinque anni (in deroga all'art. 2341bis, comma 1, c.c.), ma entro i limiti di durata del contratto per il quale è stata costituita la società. In ogni caso, al fine di "ottimizzare" la realizzazione e la gestione di più opere e servizi, anche contestuali, la società può emettere azioni correlate all'art. 2350, comma 2, c.c. o costituire patrimoni destinati 0 essere assoggettata a direzione 0 coordinamento da parte di un'altra società. L'art. 18 stabilisce particolari modalità per la quotazione di società a controllo pubblico in mercati regolamentati e l'art. 8 introduce norme per le operazioni di acquisto di partecipazioni in società già costituite; peraltro, per il Decreto Legislativo n. $175 / 2016$ è stato approvato il Decreto Legislativo correttivo 16 giugno 2017, n. 100, che introduce disposizioni integrative anche in esecuzione della sentenza n. 251 del 2016 della Corte Costituzionale, ma che non prevede modifiche rilevanti per l'art. 17.

Qualche profilo innovativo poteva derivare dall'approvazione e pubblicazione dello schema di decreto legislativo in materia di servizi pubblici locali di interesse economico generale ${ }^{41}$ che richiamava nell'art. 7 , comma 1, lett. b), tra le modalità di gestione, l'affidamento a società miste, rinviando per la scelta del socio privato al Testo Unico sulle società partecipate; il comma 6 riguardava la cessazione degli affidamenti diretti assentiti alla data del 31 dicembre 2004 a società a partecipazione pubblica già quotate in mercati regolamentati, per la scadenza prevista nel contratto di servizio con diverse modalità e conseguenze giuridiche.

L'art. 9, comma 5, lett. b) in tema di proprietà e gestione, precisava che "Qualora sia separata dalla gestione del servizio, la gestione delle reti, degli impianti e delle altre dotazioni patrimoniali essenziali, è realizzata dagli enti competenti all'organizzazione del servizio mediante: ... b) società miste, il cui socio privato sia stato scelto con procedura ad evidenza pubblica, secondo le modalità previste dal diritto dell'Unione

\footnotetext{
${ }^{40}$ v. G. CAIA, La disciplina sulle società a partecipazione pubblica, in Giorn. dir. amm., 2017, 601 ss. Per i profili societari prima del T. U. del 2016, si rinvia a F. GuerrerA, Lo statuto della nuova società "a partecipazione mista" pubblico-privata, in F. GUERRERA (a cura di), Le società a partecipazione pubblica, Giappichelli, Torino, 2010, 97 ss.

${ }^{41}$ Per l'ultimo schema di testo unico v. nota 1 ; altre norme riguardavano il contratto di servizio, le tariffe, la trasparenza, la tutela dei consumatori e la carta dei servizi (artt. 21 ss.).
} 
europea"; ulteriori disposizioni riguardavano l'Autorità per l'energia elettrica, il gas ed il sistema idrico (Aeegsi) per la nuova denominazione (Autorità di regolazione per energia, reti e ambiente, Arera), ma I'innovazione davvero rilevante era rappresentata dalla nuova competenza di regolazione per il settore dei rifiuti (art. 16), scelta che non poteva che essere confermata in un successivo intervento legislativo ${ }^{42}$. Peraltro, la mancata promulgazione del testo unico ha suscitato una significativa incertezza negli operatori del settore (enti locali e gestori nelle diverse forme), fino alla Legge di Bilancio 2018 (Legge 27 dicembre 2017, n. 205) che nell'art. 1, comma 527, ha introdotto i nuovi compiti di regolazione e controllo in materia di gestione dei rifiuti per questa Autorità, e nel comma 528 ha previsto, in effetti, la nuova denominazione di Arera.

Le disposizioni sulle società miste sono, quindi, rigidamente vincolanti e derivano in sostanza da due tendenze contrapposte: dalla nozione di socio che si ricollega ad una rilevante presenza nel contributo operativo e di capitale e dal regime di forte controllo delle società di capitali a partecipazione pubblica che inevitabilmente si estende anche alle società miste di gestione dei servizi pubblici locali. $E^{\prime}$ altresì evidente che il legislatore nazionale ha preferito fino ad oggi l'applicazione della concorrenza, limitando le società in-house a casi eccezionali (art. 16 del Decreto Legislativo n. 175/2016); vi è, quindi, un confermato indirizzo favorevole, anche in conseguenza dell'influenza della giurisprudenza e della disciplina comunitaria, per soluzioni esternalizzate di gestione dei servizi pubblici da intendersi però non in senso esclusivamente privatistico, considerata la costante previsione e diffusione delle società miste.

$\mathrm{E}^{\prime}$, inoltre, evidente la rilevante procedimentalizzazione della decisione organizzativa dell'ente locale circa il modello di gestione del servizio pubblico di rilevanza economica, a differenza della tradizionale autonomia decisionale e ciò emerge sia dal Decreto Legislativo $\mathrm{n}$. 175/2016 (ad es., art. 5 sugli oneri di motivazione analitica dell'atto deliberativo di costituzione di una società, applicabile anche nei casi di cui all'art. 17); questa tendenza emerge anche dallo schema di decreto legislativo (ritirato) per i servizi pubblici locali di interesse economico in cui è evidente il contrasto tra il principio di autonomia degli enti locali, la

42 V. Parere n. 83 del Consiglio di Stato, 17 gennaio 2017, in www.giustiziaamministrativa.it. Il parere attiene agli adempimenti in conseguenza della sentenza della Corte Costituzionale n. 251 del 2016, richiesto dalla Presidenza del Consiglio dei Ministri riguardo ad alcune questioni interpretative circa i decreti legislativi già entrati in vigore (tra questi il D.lgs. n. 175/2016); mentre per i settori per i quali la delega era nel frattempo scaduta e, in particolare, proprio per il Testo Unico in materia di servizi pubblici locali di rilevanza economica, il Consiglio di Stato ritiene possibili due soluzioni: una nuova legge di delega conforme alla sentenza della Corte Costituzionale e per I'importanza della riforma vi è I'urgenza riguardo "ai positivi effetti economici, ambientali e sociali che l'avvio della regolazione indipendente per il settore dei rifiuti avrebbe potuto, potrebbe ancora, e dovrebbe apportare al sistema Paese"; oppure la soluzione di un disegno di legge governativo che includa il contenuto del decreto legislativo delegato ritirato, dato che l'ultima versione aveva comunque già recepito i pareri delle Commissioni parlamentari. 
definizione puntuale dei procedimenti decisionali, I'esigenza di garantire la concorrenza e le forme di controllo stringenti sulle risorse utilizzate; la disciplina prevista dall'ultimo schema n. 308 del Governo si caratterizzava per varie fasi di cui quella istruttoria assai approfondita e per il coinvolgimento di soggetti esterni diversi (Arera, Autorità garante della concorrenza e del mercato) con funzione consultiva, decisionale o di controllo, con il rischio di sovrapposizioni. Questa impostazione potrebbe essere interpretata come scelta di fondo della politica dei servizi pubblici locali verso un minor carattere "locale", considerato il quadro complessivo dei soggetti e degli interessi coinvolti.

In ogni caso, la formula della società mista è confermata nella sua validità dalla costante considerazione nei processi di riforma, come in altri ordinamenti europei esaminati; nel tempo sono state modificate l'entità della partecipazione privata e le modalità di costituzione o sono stati introdotti nuovi requisiti oppure potrà essere prevista una più evidente natura collaborativa che l'avvicina maggiormente al PPPI, ma di certo questo schema rappresenta una soluzione di riferimento per gli enti locali, soprattutto in una fase di restrizione delle risorse pubbliche.

\section{LA SOCIETÀ MISTA COME ESPRESSIONE DI PPPI E LA PROMOZIONE DEL MODELLO EUROPEO IN UN CONTESTO DI CARENZA DI RISORSE FINANZIARIE E ORGANIZZATIVE DEGLI ENTI LOCALI}

La ricerca evidenzia una confermata validità dello schema giuridico della società mista di gestione di servizi pubblici locali, meglio se società quotata in borsa per le ulteriori forme di controllo, di responsabilizzazione, di trasparenza e possibilità di crescita ${ }^{43}$, soprattutto multiservizi per ragioni strategiche e considerato l'apporto finanziario e operativo all'innovazione tecnologica ${ }^{44}$; questa soluzione può essere più adeguatamente introdotta mediante il partenariato pubblico-privato incentivando l'aspetto collaborativo secondo una logica moderna, ma anche necessitata dalla crisi e dalla carenza di risorse pubbliche, organizzative e tecniche, mediante imprese competitive con dimensioni adeguate per realizzare importanti investimenti e garantire migliori standard di qualità. Ciò è in linea con la Comunicazione interpretativa ${ }^{45}$

\footnotetext{
43 Occorre precisare, tuttavia, che le attuali società di servizi quotate in borsa e strutturate attorno ad una società holding derivano da fenomeni molto particolari di aggregazione di aziende municipalizzate locali con sedi in territori contigui, risalenti ad una fase di promozione della trasformazione di aziende speciali in società per azioni (art. 115 TUEL; art. 35 della legge n. 448/2001) e di diffusione di schemi societari come espressione di una politica di privatizzazione formale generalizzata.

${ }^{44}$ Tra i programmi per l'innovazione, gli interventi di sviluppo per la nuova dimensione urbana di Smart City, v. S. ANTONIAZZI, Smart City: quadro generale di atti, programmi e competenze di livello sovranazionale, nazionale e locale, e S. VENIER, E. PIRACCINI, R. GASPARETTO, Infrastrutture, cittadini e gestione dei servizi pubblici locali, in G. F. FERRARI (a cura di), La prossima città, Mimesis, Milano, 2017, 479 ss. e 495 ss.

45 Comunicazione interpretativa della Commissione sull'applicazione del diritto comunitario degli appalti pubblici e delle concessioni ai partenariati pubblico-privati
} 
della Commissione europea del 2008 sul PPPI, che legittima le società miste e che dev'essere ancora pienamente considerata come riferimento rilevante per un'adeguata promozione, in assenza di una disciplina specifica di livello europeo e nazionale per il nostro ordinamento (e in assenza di una legge specifica sul PPP, a differenza del sistema inglese, francese e tedesco), soprattutto dopo che il Decreto Legislativo n. 50/2016 disciplina (solamente) il PPP contrattuale e l'art. 17 del Decreto Legislativo n. 175/2016 rinvia all'art. 5, comma 9, del Decreto Legislativo n. 50/2016, che richiama le società miste precisando che la scelta del privato deve derivare da una procedura ad evidenza pubblica. Il Consiglio di Stato ${ }^{46}$, invece, riconduce da anni e con convinzione le società miste al PPPI di formulazione europea, forse non particolarmente nota al legislatore. Tuttavia, anche la Comunicazione del 2008 deve essere interpretata con un contenuto ormai evoluto e di immediata applicabilità nel nostro ordinamento; infatti, non solo l'apporto del privato operatore economico alla società mista deve consistere nel conferimento di capitali (un socio solo finanziatore sarebbe comunque un'ipotesi teorica) o di altri beni (la struttura aziendale), nella partecipazione attiva all'esecuzione dei compiti affidati alla società e/o nella gestione dell'attività, ma anche nell'adozione di atti di indirizzo (indicazione di obiettivi per piani industriali realizzabili, designazione dell'amministratore delegato, formalmente diverso dal socio privato) e nella regolazione dei rapporti societari interni per la fase di esecuzione mediante disposizioni dello Statuto che richiamino il PPPI, anche nei rapporti con i terzi. Altrettanto limitata appare la definizione di socio pubblico nel solo ruolo di controllore delle operazioni in relazione alla partecipazione azionaria e agli organi decisionali, dato che sarebbe necessaria un'effettiva partecipazione alla funzione di indirizzo per il piano industriale, in quanto socio destinatario della medesima disciplina societaria, dei relativi diritti, obblighi e responsabilità a cui si aggiungono la tutela di interessi pubblici ed il regime di concorrenza.

Del resto, il coinvolgimento del privato nel partenariato istituzionalizzato è giustificato e necessitato da esigenze concrete, espressione della logica pragmatica anglosassone da cui deriva; I'obiettivo più che mai pressante degli enti pubblici è quello di reperire le risorse necessarie nel settore privato per assicurare la fornitura del servizio pubblico, nel senso di capitali, conoscenze tecniche, scientifiche, organizzative già sperimentate per garantire un'azione amministrativa efficiente e così "alleggerire" gli oneri economico-finanziari. Tuttavia, non

istituzionalizzati (PPPI), (2008/C 91/02), cit.; la Commissione, come è noto, equipara il PPP al PPPI per l'applicazione del diritto degli appalti e delle concessioni.

46 Si rinvia a Consiglio di Stato, parere, Sez. II, 18 aprile 2007, n. 456; Consiglio di Stato, Adunanza Plenaria, 3 marzo 2008, n. 1, che esamina in modo approfondito la qualificazione della società mista come applicazione di PPPI secondo il Libro verde e la Comunicazione della Commissione del 2008; Consiglio di Stato, Sez. V, 10 settembre 2014, n. 4599; Consiglio di Stato, Sez. V, 22 gennaio 2015, n. 257; il parere e le sentenze del Consiglio di Stato sono reperibili nel sito istituzionale www.giustiziaamministrativa.it. 
si tratta di una fase anticipata di privatizzazione, ma di una soluzione alternativa autonoma di effettiva collaborazione tra pubblica amministrazione e privati imprenditori (socio tecnico e in parte finanziatore, scelto con procedura ad evidenza pubblica) mediante un'entità giuridica detenuta congiuntamente, con effetti positivi per la collettività; quindi, la società mista dev'essere intesa come modalità organizzativa che consente all'ente locale di controllare l'erogazione del servizio, ma con poteri ampi in qualità di socio per l'esercizio dell'attività e l'erogazione delle prestazioni. $E^{\prime}$, peraltro, evidente una certa "fungibilità" tra appalto e società mista per la gestione del servizio, ma la seconda soluzione garantisce - almeno in teoria - un maggiore coinvolgimento dell'ente pubblico socio e del socio imprenditore in una struttura organizzativa stabile con competenze e conoscenze tecniche adeguate per I'attività di erogazione di servizi pubblici; in realtà, la società mista consente all'ente locale di "sorvegliare" meglio la gestione del partner privato, rispetto al rapporto che deriva dal contratto d'appalto.

Lo schema di base ancora attuale, secondo l'impostazione generale della Commissione europea, si riferisce alla "costituzione di una nuova impresa il cui capitale è detenuto congiuntamente dall'amministrazione aggiudicatrice e dal partner privato (in alcuni casi, da più amministrazioni aggiudicatrici e/o partner privati), e nell'aggiudicazione di un appalto pubblico o di una concessione a tale entità a capitale misto di nuova costituzione oppure nella partecipazione di un partner privato a un'impresa pubblica già esistente che esegue appalti pubblici o concessioni" ${ }^{\prime 47}$. La procedura ad evidenza pubblica è decisiva per la selezione del partner privato con l'apporto del contributo operativo all'esecuzione delle prestazioni e finanziario e/o alla gestione della società mista, e per l'aggiudicazione ad essa dell'appalto pubblico o della concessione del servizio pubblico, con differenze rilevanti ovviamente quanto al trasferimento del rischio. Da ciò deriva l'esigenza di definire nello statuto una peculiare regolamentazione dei rapporti tra i soci per gli aspetti proprietari, il governo della società e l'esercizio dell'impresa comune, aspetti che un contratto di servizio non potrebbe regolare adeguatamente; anche la concessione già realizza una forma contrattuale di collaborazione pubblico-privata, ma alla luce di una dinamica di ruoli più tradizionale in senso pubblicistico, e assai diffusa per l'efficacia pratica e la più approfondita disciplina del rischio con il Decreto Legislativo n. 50/2016 e la Direttiva (UE) n. 23/2014.

\section{LE CONCLUSIONI DELL'INDAGINE}

La società mista si caratterizza per una dinamica di rapporti in continua tensione in relazione ad interessi diversi (I'interesse della parte pubblica alle migliori prestazioni di servizio pubblico e l'interesse del socio privato agli utili) che possono essere anche mediati per una reciproca

\footnotetext{
47 V. Comunicazione interpretativa della Commissione (2008/C 91/02), cit.; Risoluzione del Parlamento europeo sul Libro verde (COM (2004) 327 del 30 aprile 2004) sui servizi di interesse generale (P5_TA (2004)0018).
} 
positiva influenza ed interazione; inoltre, l'ente locale socio è pienamente inserito nello schema societario, a differenza del caso dell'esternalizzazione del servizio, ed è destinatario di norme, di obblighi, di diritti e di strumenti del regime societario comune che consentono di controllare e incidere sulla gestione, sulle modalità di erogazione per garantire la qualità delle prestazioni in quanto ente rappresentativo di interessi pubblici ed interlocutore delle richieste dei cittadini-utenti. Ovviamente l'ente locale dovrebbe essere un socio competente per gli aspetti tecnici di base, presupposto in realtà non scontato.

Il Decreto Legislativo n. 175/2016, oltre a stabilire nell'art. 17 la quota di partecipazione del socio privato non inferiore al trenta per cento con ampie possibilità di combinazione anche in forma di partecipazione pubblica minoritaria (si consideri anche la recente tendenza degli enti locali a vendere le azioni riducendo la quota di partecipazione per far fronte ad esigenze di bilancio), prevede l'applicabilità come regola generale di diversi strumenti privatistici con possibilità di alcune deroghe, e, quindi, consente ad entrambe le parti un esercizio adeguato dei diritti di socio con le relative responsabilità; peraltro, si pone il problema di tutelare adeguatamente i diritti del socio privato, al fine di attrarre maggiore interesse alla partecipazione societaria, anche per gli operatori economici di società già costituite prima del T. U. Tuttavia, occorre chiedersi se questo "modello" che recepisce regole già note (anche per il contributo della giurisprudenza) ed introduce alcune innovazioni, sia in concreto realizzabile con risultati positivi, considerati, ad es., i vincoli di attività, gli oneri procedimentali e di motivazione analitica di cui agli artt. 2, 5 del Decreto Legislativo n. 175/2016, ma soprattutto nell'attuale situazione economica. Peraltro, la realtà delle società miste multiservizi di grandi dimensioni strutturate in forma di "gruppo" in una società holding, derivano da processi di aggregazione perfezionati in un contesto assai diverso anche per le autonomie locali, in assenza degli attuali vincoli normativi di fonte comunitaria e nazionale e di condizioni economiche sfavorevoli; quindi, si tratta, di esperienze di fatto difficilmente attuabili con la stessa dinamica.

Come emerge dalla ricerca l'adozione del partenariato pubblicoprivato è diffuso negli ordinamenti europei, soprattutto in un periodo di crisi finanziaria ed economica, nonostante casi di rimunicipalizzazione per alcuni servizi pubblici esternalizzati per un certo periodo (scelta attuata in Germania e in fase di accesa discussione per il servizio idrico in Spagna). L'esame del percorso di alcune esperienze evidenzia obiettivi comuni, ma con diverse tecniche di soluzione che in parte derivano da tradizioni nazionali differenziate. La disciplina della società mista negli ordinamenti osservati dipende anche dalla presenza o meno di una legge generale nazionale sul PPP, introdotta prima della Comunicazione del 2008; in Francia è evidente una certa creatività del legislatore per la tradizione del service public e le diverse soluzioni alternative di partecipazione pubblicoprivata nel settore dei servizi pubblici, mentre l'ordinamento spagnolo si caratterizza per un percorso assai simile al caso italiano in conseguenza di veloci misure di razionalizzazione della spesa pubblica e delle funzioni 
degli enti locali, e per una significativa diffusione delle società miste. Del tutto unica l'esperienza del Regno Unito per la tradizione del PPP e del PFI (di recente ulteriormente promosso in relazione al partenariato per l'innovazione e al recepimento delle direttive (UE) n. 23 e n. 24 del 2014) e I'utilizzo della società mista in settori diversi dai servizi pubblici locali come considerati nella maggioranza degli ordinamenti europei, per la risalente e consolidata privatizzazione; peraltro, è singolare il veloce recepimento delle direttive del 2014 sui contratti pubblici in una fase di dibattuto referendum per l'uscita dall'UE. Per la Germania occorre, invece, ricordare la recente tendenza di ritorno alla gestione totalmente pubblica (soluzione risalente per il ruolo delle collettività locali e dei loro enti), dopo anni di diffusa gestione privata che non ha suscitato valutazioni complessivamente positive e per una consentita ampia partecipazione dei cittadini-utenti alla scelte di politica dei servizi pubblici locali; peraltro, la legge generale sul PPP è stata applicata anche in settori diversi per sostenere la ripresa economica.

I diversi sistemi presentano dei tratti comuni: la società mista si colloca inevitabilmente tra il diritto amministrativo e il diritto privato delle società, tra disciplina speciale di settore e disciplina generale con soluzioni operative tipicamente pubblicistiche (gara pubblica per la selezione del socio privato, controlli sugli atti e sull'attività), occasioni di "conflitto" tra regole e dubbi interpretativi. I principi comuni derivano dal diritto e da atti di soft law dell'UE e con questi presupposti essenziali è possibile riconoscere un modello europeo secondo una logica più ampia volta all'integrazione rispetto alle esigenze contingenti di ogni singolo ordinamento; nel tempo, gli ordinamenti nazionali hanno recepito direttive e attuato norme dell'UE, adottando soluzioni di adeguamento a pronunce della Corte di Giustizia nonché misure di riduzione della spesa pubblica anche in conseguenza di sollecitazioni da parte di istituzioni europee. Esiste, quindi, una certa uniformità in cui inserire un modello comune con alcune particolarità che dipendono dall'ambito di consentita discrezionalità nazionale.

La difficoltà di ricostruire un "modello italiano" innovativo in assenza di una strategia più originale e non limitata al solo "riordino" di norme stratificate nel tempo, potrebbe essere superata proprio con un più deciso riferimento al modello europeo di PPPI inserito nell'attuale contesto dell'economia con misure di promozione e un sistema di incentivi concreti per attrarre gli operatori economici privati. Nel nostro ordinamento il quadro giuridico è attualmente in una fase transitoria di parziale riforma con molte incertezze ${ }^{48}$, dato che occorre tener conto di alcune innovazioni che riguardano gli enti territoriali (possibili soci di maggioranza o di minoranza), dell'attuazione non semplice del T. U. sulle società a partecipazione pubblica criticabile per alcuni aspetti e, in particolare, per l'eccessiva procedimentalizzazione delle scelte degli enti locali e il

\footnotetext{
48 Per alcune considerazioni assai critiche sulle riforme introdotte e in corso e sulla loro autenticità, in base alla legge delega n. 124/2015, si rinvia a F. MERUSI, L' "imbroglio" delle riforme amministrative, Mucchi, Modena, 2016, 30 ss.
} 
coordinamento, non agevole per alcuni profili, con il Decreto Legislativo n. 50/2016; inoltre, il Decreto Legislativo n. 175/2016 è stato elaborato anche in collegamento con lo "sfortunato" T. U. sui servizi pubblici locali, che avrebbe comunque comportato ulteriori perplessità per l'impostazione "centralista" non del tutto rispettosa delle autonomie, confermata nell'ultima versione dello schema approvato dal Consiglio dei Ministri e poi ritirato.

In conclusione, la ricostruzione del quadro normativo attuale sulle società miste coinvolge il $\mathrm{T}$. U. sulle società a partecipazione pubblica, la disciplina vigente sui servizi pubblici che include il TUEL per le pochissime norme ancora in vigore, le definizioni del diritto dell'UE di servizio di interesse economico generale e di interesse generale ${ }^{49}$, le diverse discipline speciali ormai prevalenti per la tecnicità dei settori nonché le disposizioni del Decreto Legislativo n. 50/2016 in materia di contratti pubblici, le leggi regionali settoriali in tema di partecipazioni societarie e di servizi per alcune Regioni, le norme di riordino delle funzioni amministrative delle autonomie e la regolazione dell'Arera e dell'Autorità garante della concorrenza e del mercato: un panorama complesso che non può che suscitare preoccupazione negli enti locali intenzionati ad istituire una società mista o ad acquisire una quota in una società privata oppure ad introdurre alcune modifiche nel regime di una società mista esistente o a dismettere anche in parte una partecipazione (forse più probabile, considerati i disavanzi di bilancio) considerato l'intricato panorama di controlli, adempimenti e (doverose) responsabilità. A ciò si aggiunge il compito non agevole di individuare legittimamente un servizio pubblico locale in caso di nuova gestione, in assenza di specifici criteri di riferimento espressi in una legge nazionale e considerata la nozione europea di servizio di interesse economico generale.

La preoccupazione si estende ovviamente agli operatori economici, che tendono ragionevolmente a confidare in regole certe e chiare per un'efficace programmazione industriale in un contesto di persistente incertezza dell'evoluzione dell'economia.

\section{BIBLIOGRAFIA}

ABBADESSA P., Le società miste per i servizi locali: profili organizzativi speciali, in TRIMARCHI F. (a cura di), Le società miste per i servizi locali, Giuffrè, Milano, 1999, 87 ss.

Anselmi D., L'affidamento a società miste, in Vigneri A., Sebastiani M. (a cura di), Società pubbliche e servizi locali, Astrid, 2016, in www.astridonline.it.

ANTONIAZZi S., La privatizzazione del servizio idrico in Inghilterra e in Galles e la riforma Water Act 2014, in MidiRi M., ANTONIAZZI S. (a cura di), Servizi pubblici locali e regolazione, Editoriale Scientifica, Napoli, 2015, 255 ss.

${ }^{49}$ Si rinvia allo studio collettaneo di V. Parisio, V. Aguado I Cudolà, B. Noguera De LA Muela (dir.), Servicios de interés general, colaboración público-privada y sectores específicos, Tirant lo blanch, Valencia, Giappichelli, Torino, 2016. 
Antoniazzi S., Società miste e servizi pubblici locali. Esperienze nazionali e modello europeo, Editoriale Scientifica, Napoli, 2017

Antoniazzi S., Smart City: quadro generale di atti, programmi e competenze di livello sovranazionale, nazionale e locale, in G. F. FERrARI (a cura di), La prossima città, Mimesis, Milano, 2017, 479 ss.

Bifulco R., L'onda lunga della sentenza 251/2016 della Corte Costituzionale, in www. federalismi.it, 8 febbraio 2017

CAIA G., La disciplina sulle società a partecipazione pubblica, in Giorn. dir. amm., 2017, 601 ss.

Cammelli M., I servizi pubblici dell'amministrazione locale, in Le Regioni, 1992,24 ss.

CAMmelli M., Ziroldi A., Le società a partecipazione pubblica nel sistema locale, Maggioli, Rimini, 1997

Cammelli M., Concorrenza, mercato e servizi pubblici: le due riforme, in Riv. trim. app., 2003, 517 ss.

Caruso G. M., Il socio pubblico, Jovene, Napoli, 2016

ChItI M. P., Verso la fine del modello di gestione dei servizi pubblici locali tramite società miste, in Foro amm. TAR, 2006, II, $1161 \mathrm{ss.}$

DalL'Osso C., Una società verso il futuro, I servizi pubblici locali nella prospettiva di Hera, La Mandragora, Imola, 2009

DE PRETIS D., Servizi pubblici locali e società miste: una visione comparativa, in ChITI M. P. (a cura di), Le forme di gestione dei servizi pubblici locali tra diritto europeo e diritto locale, il Mulino, Bologna, 2006, 104 ss.

DonAtivi V., Le società a partecipazione pubblica, Wolters Kluwer, Assago, 2016

Dugato M., Le società per la gestione nei servizi pubblici locali, Il Giornale di diritto amministrativo, Quaderni, Ipsoa, Milano, 2001

Dugato M., Il servizio pubblico locale: incertezze qualificatorie e tipicità delle forme di gestione, in Giorn. dir. amm., 2002, 24 ss.

DugAto M., Le società degli enti territoriali alla luce dell'art. 13 del D. L. n. 223/2006, in Cammelli M., Dugato M. (a cura di), Studi in tema di società a partecipazione pubblica, Giappichelli, Torino, 2008, 347 ss.

Dugato M., Il partenariato pubblico-privato: origine dell'istituto e sua evoluzione, in MAStRagostino F. (a cura di), La collaborazione pubblico-privato e l'ordinamento amministrativo, Giappichelli, Torino, 2011, 55 ss.

Dugato M., Le società a partecipazione mista per la gestione dei servizi pubblici locali. Il procedimento di costituzione, l'affidamento dei lavori e la relazione tra ente socio e società, in DugATo M., MASTRAgostino F. (a cura di), Partecipazioni, beni e servizi pubblici tra dismissioni e gestione, Bononia University Press, Bologna, 2014, 213 ss.

DugAto M., Le società a partecipazione pubblica, in Giorn. dir. amm., 2013, 855 ss.

GUERRERA F., Lo statuto della nuova società "a partecipazione mista" pubblico-privata, in GUERRERA F. (a cura di), Le società a partecipazione pubblica, Giappichelli, Torino, 2010, 97 ss. 
MARCHESI D., Riordino delle partecipate degli enti locali, criticità, possibili policy, interventi in atto, in Analisi giuridica dell'economia, 2015, 335 SS.

MAROTTA S., La spending review nei servizi pubblici locali: necessità di razionalizzare, volontà di privatizzare, in Munus, 2014, 261 ss.

MARRA A., La razionalizzazione delle società partecipate dagli enti locali dopo la legge di stabilità 2015, in Dir. econ., 2015, 299 ss.

MAZZARELli M., La società per azioni con partecipazione comunale, Giuffrè, Milano, 1987

Merusi F., voce Servizio pubblico, in Nuoviss. Dig. Ital., XVII, 1970, 215 SS.

Merusi F., Servizi pubblici instabili, il Mulino, Bologna, 1990

MERUSi F., Il sogno di Diocleziano. Il diritto delle crisi economiche, Giappichelli, Torino, 2013

Merusi F., L' "imbroglio" delle riforme amministrative, Mucchi, Modena, 2016

MoRbidelli G., Società miste, servizi pubblici e opere accessorie, in Riv. trim. appalti, 1997, 493 ss.

MoRbidelli G., I controlli sulle società a partecipazione pubblica, in G. MoRBIDelLI, Scritti di diritto dell'economia, Giappichelli, Torino, 2001, 279 ss. e in Predieri A. (a cura di), Controlli societari e governo dell'impresa, Giappichelli, Torino, 1999, 99 ss.

Occhilupo R., G. RomA, Le società miste affidatarie di servizi pubblici locali: un'analisi degli assetti di governo, in Analisi giuridica dell'economia, 2015, 496.

Parisio V., Aguado i Cudolà V., Noguera de la Muela B. (dir.), Servicios de interés general, colaboración público-privada y sectores específicos, Tirant lo blanch, Valencia, Giappichelli, Torino, 2016

PIPERATA G., Tipicità e autonomia dei servizi pubblici locali, Giuffrè, Milano, 2005

PIPERATA G., Le società a partecipazione pubblica nella gestione dei servizi degli enti locali, in Cammelli M., Dugato M. (a cura di), Studi in tema di società a partecipazione pubblica, Giappichelli, Torino, 2008, 312 ss. a cura di

PiPERATA G., La trasformazione delle società a partecipazione pubblica. Dalla società in house alla società a partecipazione mista, in DUGATO M., Mastragostino F. (a cura di), Partecipazioni, beni e servizi pubblici tra dismissioni e gestione, cit., 151 ss.

PIPERATA G., Liberalizzazioni e interesse generale: il caso del servizio idrico integrato, in Diritti Lavori Mercati, 2015, II, 6 ss.

PIPERATA G., Tendencias recientes en las reformas de la normativa italiana sobre los servicios públicos locales: de la remunicipalización hacia la desmunicipalización, in Font i Llovet T., DíEz SÁnchez J. J. (Coords.), Los servicios públicos locales. Remunicipalización y nivel óptimo de gestión, Iustel, Madrid, 2017, 221 ss.

PIPERATA G., La partecipazione delle pubbliche amministrazioni a società di capitali nel nuovo disegno di riforma: modelli, procedure e limiti, in 
Cerioni F. (a cura di), Le società pubbliche nel Testo Unico, Giuffrè, Milano, 2017, 1 ss.

Poggi A., G. Boggero, Non si può riformare la p. a. senza intesa con gli enti territoriali: la Corte Costituzionale ancora una volta dinanzi ad un Titolo V incompiuto, in www.federalismi.it, 28 dicembre 2016

SABLONE S., Art. 17. Società a partecipazione mista pubblico-privata, in Meo G., Nuzzo A. (diretto da), Il Testo Unico sulle società pubbliche, Cacucci, Bari, 2016, 249 ss.

SAntiago Iglesias D., Las sociedades de economía mixta come forma de gestión de los servicios públicos locales, Madrid, Iustel, 2010

Santiago Iglesias D., Las sociedades municipales, in Almeida CerReda M., Tubertini C., Costa Gonçalves P. (dirs.), Santiago Iglesias D., Di LASCIO F., P. CRUZ Silva (coords.), La racionalización de la organización administrativa local: las experiencias española, italiana y portuguesa, Civitas, Thomson Reuters, Navarra, 2015, 230 ss.

Santiago Iglesias D., Empresas locales, in F. Velasco Caballero (dir.), Tratado de derecho económico local, Marcial Pons, Madrid, 2017, 171 SS.

SCHMITT C., The diffusion of privatisation in Europe: political affinity or economic competition?, in Public Administration, 2014, 615 ss.

SORACE D., Pubblico e privato nella gestione dei servizi pubblici locali mediante società per azioni, TRIMARCHI F. (a cura di), Le società miste per i servizi locali, cit., 135 ss.

TUBERTINI C., L'attuazione regionale della legge 56/2014: verso un nuovo assetto delle funzioni amministrative, in Le Regioni, 2016, 99 ss.

URSI R., Società ad evidenza pubblica, Editoriale Scientifica, Napoli, 2012, ristampa 2013

URSI R., Il governo del gruppo pubblico locale al tempo della Spending review, in PASSALACQUA M. (a cura di), Il <<disordine>> dei servizi pubblici locali, Giappichelli, Torino, 2015, 145 ss.

VAlAGUZZA S., Società miste a partecipazione comunale, Giuffrè, Milano, 2012

VAndelli L., Gardini G., Tubertini C. (a cura di), Le autonomie territoriali: trasformazioni e innovazioni dopo la crisi, Maggioli, Rimini, 2017

VENIER S., Le prospettive strategiche e gestionali dei servizi pubblici locali in Italia, in Midiri M., ANToniazzi S. (a cura di), Servizi pubblici locali e regolazione, cit., 80 ss.

Venier S., Piraccini E., Gasparetto R., Infrastrutture, cittadini e gestione dei servizi pubblici locali, in FerRARI G. F. (a cura di), La prossima città, cit. , 495 ss.

Ziruolo A., Valore pubblico e società partecipate. Tendenze evolutive della performance, Franco Angeli, Milano, 2016 